\title{
High CHI3L1 expression is associated with glioma patient survival
}

\author{
Giedrius Steponaitis, Daina Skiriutè, Arunas Kazlauskas, leva Golubickaitė, Rytis Stakaitis, \\ Arimantas Tamašauskas and Paulina Vaitkiené*
}

\begin{abstract}
Background: Survival of glioma patients with the same tumor histology and grade can vary significantly, and some low-grade gliomas transform to a more malignant phenotype. There is a need of molecular signatures, which are better predictors of the patient diagnosis, outcome of treatment, and prognosis than the diagnosis provided by histopathology. We propose CHI3L1 mRNA expression as a prognostic biomarker for patients with glioma.

Methods: We measured CHI3L1 expression with quantitative real time-polymerase chain reaction (qRT-PCR) in the cohort of 98 patients with different grade glioma: 10 grade I pylocytic astrocytomas, 30 grade II diffuse astrocytomas, 20 grade III anaplastic astrocytomas, and 38 grade IV astrocytomas (glioblastomas). Statistical analyses were conducted to investigate the association between CHI3L1 mRNA expression levels and patient clinical variables.

Results: We demonstrated that mRNA expression of $C H I 3 L 1$ was evidently higher in glioblastoma than in lower grade glioma tissues. We evaluated correlations between CHI3L1 expression, clinicopathological characteristics, and the outcomes of the patients. Patients with high CHI3L1 expression had a shorter overall survival $(p<0.001)$.

Conclusions: Findings presented in our study showed that increased mRNA level of CHI3L 1 could be associated with the progression of astrocytoma and poor patient survival not only for glioblastoma, but for lower grade astrocytoma tumors as well. Further investigation will be required to evaluate CHI3L1 value as a molecular marker for astrocytoma prognoses and for novel treatment strategies against all grade astrocytomas.
\end{abstract}

Keywords: CHI3L1, Chitinase 3-like 1, YKL-40, Glioma, Astrocytoma, Survival, mRNA

\section{Background}

Brain tumours are classified according to the WHO classification of CNS tumours (2007 which is based on histological characteristics of the tumour) [1]. The previous works demonstrated that molecular signatures allow a better characterisation of the pathology than the current clinical scheme based on histopathological classification [2, 3]. According to the WHO classification, gliomas are subdivided into the (rare) ependymomas, oligodendrogliomas, and astrocytomas, which are the largest group of gliomas [4]. Astrocytoma has a well differentiated variant, known as pilocytic astrocytoma (WHO grade I, diffuse astrocytoma (WHO grade II), anaplastic astrocytoma (WHO grade III) and astrocytoma grade IV, which is known as glioblastoma

\footnotetext{
*Correspondence: paulina.vaitkiene@gmail.com

Laboratory of Neurooncology and Genetics, Neuroscience Institute, Medical

Academy, Lithuanian University of Health Sciences, Eiveniu str. 4, Kaunas LT-50009, Lithuania
}

(c) 2016 Steponaitis et al. Open Access This article is distributed under the terms of the Creative Commons Attribution 4.0 International License (http://creativecommons.org/licenses/by/4.0/), which permits unrestricted use, distribution, and reproduction in any medium, provided you give appropriate credit to the original author(s) and the source, provide a link to the Creative Commons license, and indicate if changes were made. The Creative Commons Public Domain Dedication waiver (http://creativecommons.org/publicdomain/zero/1.0/) applies to the data made available in this article, unless otherwise stated.

multiforme. Glioblastoma is one of the most malignant forms of human brain tumour. Glioblastoma is clinically classified as primary or secondary subtypes depending on whether it was diagnosed as a de novo tumor or it derived from gliomas of lower grade, respectively [1]. However, survival of glioma patients with the same tumor histology and grade can vary significantly, and some low-grade gliomas transform to a more malignant phenotype [5]. There is need of molecular signatures, which are a better predictor of the patient diagnosis, outcome of treatment, and prognosis than the diagnosis provided by histopathology.

Chitinase 3-like 1 (CHI3L1) plays a role in cell proliferation, differentiation, apoptosis, angiogenesis, inflammation and extracellular tissue remodeling [6]. CHI3L1 is located on chromosome 1q32.1, and the product, YKL-40, a 40$\mathrm{kDa}$ glycoprotein, is secreted by numerous human cells such as cartilage, synovium, endothelial cells, inflammatory 
cells, and cancer cells [7]. There are data that, presence of YKL-40 protein in serum, could be a prognostic predictor of glioblastoma [5, 8]. The high serum levels of the glycoprotein are associated with poor prognosis of various medical, inflammatory and tumour processes [9-12]. These medical, inflammatory, and malignant diseases all possibly contribute to the levels of serum YKL-40. In our study, we used real-time quantitative PCR (qRT-PCR) to measure the quantitative expression of CHI3L1 in different grade astrocytoma tissues without the influence of other malignancies or medical diseases.

\section{Methods}

\section{Patients and tissue samples}

In total 98 post-operative samples obtained from patients diagnosed with different malignancy grade gliomas were analyzed: 10 grade I pylocytic astrocytomas, 30 grade II diffuse astrocytomas, 20 grade III anaplastic astrocytomas, and 38 grade IV astrocytomas (glioblastomas). All glioma tumor samples were collected in Neurosurgery Clinics of Hospital $(\mathrm{NCH})$ of Lithuanian University of Health Sciences (Kaunas, Lithuania) during the period from the year 2003 to 2014 with informed consent from patients. Tumor samples were collected, following written informed consent, in accordance with the Lithuanian regulations and the Helsinki Declaration. Written informed consent was obtained for every patient under the approval of the Ethics Committee, Lithuanian University of Health Sciences. Database closure was in March 2015. Diagnoses were established by pathologists at the $\mathrm{NCH}$ according to the World Health Organization (WHO) classification. Glioma samples were stored in liquid nitrogen until analysis. The following clinical data were collected for each patient: age at the time of the operation, gender, and patient status. The overall survival of the patient was calculated from the date of the operation to the date of death or the last recorded contact with the live patient (censored). None of the patients had received chemotherapy or radiation before surgery.

\section{Methylation specific PCR}

Brain tumor tissue specimens after dissection were snap-frozen in liquid nitrogen and stored until analysis. Tumor DNA was purified from 50-100 mg of frozen tissue using ZR Genomic DNA Tissue MiniPrep (Zymo Research) according to manufacturer's protocol. The concentration of DNA was measured using NanoDrop 2000 Spectrophotometer (Thermo Scientific) before DNA bisulfite-treatment. Obtained values were used in subsequent phases of the study as approximate quantity. Methylation status of $C H I 3 L 1$ gene promoter was determined by bisulfite treatment of DNA. $400 \mathrm{ng}$ of total genomic DNA was modified using EZ DNA Methylation Kit (Zymo Research). Bisulfite-treated DNA was eluted in
$40 \mu \mathrm{l}$ nuclease-free water, and stored in $-80{ }^{\circ} \mathrm{C}$ until analysis. "Human brain DNA" (Zymo Research, Cat. No. D5018) served as a normal brain tissue control. For negative methylation control normal human blood lymphocyte DNA treated with bisulfite was used. Bisulfite Converted Universal Methylated Human DNA Standard (Zymo Research) was used as a positive control for DNA methylation. Promoter methylation was detected by methylation-specific PCR (MSP). Each MSP reaction incorporated approximately $20 \mathrm{ng}$ of bisulfite-treated DNA as template. Specific primers for methylated and unmethylated target DNA sequence were designed using free access online software's (1-3). In total two primer sets for different $\mathrm{CpG}$ dinucleotides sites were used for the study. MSP primers for methylated CHI3L1 were:

$1^{\text {st }}: 5^{\prime}-\quad$ TTTTTATAAAAGGGTTGGTTTGTC - $3^{\prime}$ (sense)

5' - TAACCCAAATACCTATTTAAAACGC-3' (antisense)

$2^{\text {nd }}$ : 5' $^{\prime}$ TGTTAGATGTTCGTGTAGTCGTTTC-3' (sense)

5' - CCAAAAATACTTTAAACCCCGAT-3' (antisense)

and for unmethylated sequence:

$1^{\text {st }}: \quad 5^{\prime}-\quad$ TTTTTATAAAAGGGTTGGTTTGTTG-3 (sense)

5' - AACCCAAATACCTATTTAAAACACC -3' (antisense)

$2^{\text {nd }}: 5^{\prime}$ - TTAGATGTTTGTGTAGTTGTTTTGT -3' (sense)

5' - CCAAAAATACTTTAAACCCCAAT -3' (antisense)

Reaction was performed in $15 \mu \mathrm{l}$ of total volume by using 7.5 $\mu \mathrm{l}$ Maxima Hot Start PCR Master Mix (Thermofisher Scientific) with Hot start Taq DNA polymerase and 10 pmol of each primer (Metabion International AG). MSP was performed for 36 cycles with the reaction starting at $95^{\circ} \mathrm{C}$ for $15 \mathrm{~s}$., annealing of $58{ }^{\circ} \mathrm{C}$ and $61^{\circ} \mathrm{C}\left(1^{\text {st }}\right.$ and $2^{\text {nd }}$ primer pair respectively) for $30 \mathrm{~s}$., and extension of $72{ }^{\circ} \mathrm{C}$ for $20 \mathrm{~s}$. Amplification products were analyzed on $2 \%$ agarose gels with ethidium bromide (final conc. $0.05 \mu \mathrm{g} / \mathrm{ml}$ ) and documented under UV gel imaging system (Gel Doc XR+ System, BioRad). The presence of a PCR product of the correct molecular weight indicated the presence of either unmethylated or methylated alleles. In case of appearance of both unmethylated and methylated signals, case was considered as being methylated.

RNA extraction, CDNA synthesis and quantitative RT-PCR Total RNA from cryogenically homogenized tumor tissue was purified using TRIzol Reagent (Ambion, Life Technologies). To increase the yield of RNA, homogenate was additionally sonicated using ultrasound (500-W ultrasonic processor, Cole Parmer). The concentration of 
RNA was measured using NanoDrop 2000 Spectrophotometer (Thermo Scientific). Obtained values were used in subsequent phases of the mRNA quantitation as approximate quantity. Reverse transcription (RT) was carried out using RevertAid H Minus M-MuLV Reverse Transcriptase (Thermofisher Scientific) and random hexamer primers (Thermofisher Scientific) in a total reaction volume of $20 \mu \mathrm{l}$ according to the manufacturer's protocol. For inhibition of mRNA degradation RiboLock RNase inhibitor (ThermoFisher Scientific) was used. After synthesis cDNA stock was stored at $-80{ }^{\circ} \mathrm{C}$. CHI3L1 mRNA expression was analyzed using quantitative real-time RT-PCR TaqMan probe assay in 3 replicates on 7500 Fast Real-time PCR detection system (Applied Biosystems) and Relative Quantitation method $(\triangle \mathrm{CT})$. Reactions have been assembled into a total volume of $12 \mu \mathrm{l}$ of each, which included: $15 \mathrm{ng}$ of the cDNA, $6 \mu \mathrm{l}$ of TaqMan Universal Master Mix II, no UNG (Applied biosystems) with AmpliTaq Gold ${ }^{\circledR}$ DNA Polymerase and Taqman expression probe for CHI3L1 (assay no: Hs00609691_m1) and nuclease-free water. $\beta$ actin mRNA expression was analyzed using quantitative real-time RT-PCR SYBR Green I assay on the same detection system. $12 \mu \mathrm{l}$ of reaction mix consist of $15 \mathrm{ng}$ of the cDNA, $6 \mu \mathrm{l}$ Maxima Hot Start PCR Master Mix (Thermofisher Scientific) with Hot start Taq DNA polymerase, primers for $\beta$-actin (5' -AGAGCTACGAGCTGCCTGAC-3' (sense) and $5^{\prime}$-AGCACTGTGTTGGCGTACAG-3' (antisense), amplicon length: $184 \mathrm{bp}$ to a total concentration of $0.1 \mu \mathrm{M}$, and nuclease-free water. PCR has been carried out for 40 cycles consisting of $95{ }^{\circ} \mathrm{C}$ for 30 s., $60{ }^{\circ} \mathrm{C}$ for $30 \mathrm{~s}$., and $72{ }^{\circ} \mathrm{C}$ for $30 \mathrm{~s}$. Fluorescent data were converted to threshold cycle $(\mathrm{CT})$ measurements. $\triangle \mathrm{CT}$ values were calculated from averaged replicates $\mathrm{CT}$ according to the formula $\Delta \mathrm{CT}=\mathrm{CT} C H I 3 L 1-\mathrm{CT} \beta$-actin. Differences between the plates were equalized using reference sample (Human normal brain) Ct values realignment between experiments. To be able to quantify samples in $95 \%$ of cases, samples with standard deviation more than 0.25 (Ct between replicates) were eliminated from analysis. The final result was given as $\log 2$ of $2^{-(\Delta C T)}$ calculation. $\mathrm{Hu}$ man normal brain RNA sample "FirstChoice Human Brain Reference RNA" (Ambion), which was a pool of RNAs assembled from multiple donors from several brain regions, as described by the manufacturer, served as a control sample for standard curve design for $\beta$-actin.

\section{Statistical analysis}

SPSS Statistics 22 (SPSS Inc., Chicago, IL) software package was used for statistical analysis. Association between CHI3L1 mRNA level data and clinical features of glioma patients were analyzed by Chi-Square Test. The KaplanMeier method was used to estimate survival functions. For comparing survival time distribution between groups the log-rank test was used. Prognostic factors such as gender, age, pathological grade, CHI3L1 promoter methylation and mRNA expression were first examined individually (univariate analysis), and all factors that had strong impact on survival $(p<0.05)$ were then evaluated jointly in Cox regression analysis (multivariate analysis). Differences in CHI3L1 mRNA expression between different glioma malignancy grades were evaluated using the One-way ANOVA analysis. The level of significance was set to $p<0.05$.

\section{Results}

Analyses of CHI3L1 expression in different grade gliomas At first we checked whether the CHI3L1 mRNA level is associated with the different histopathological grade of glioma. One-way ANOVA analysis showed significant $m$ RNA level differences among tumor malignancy groups $(\mathrm{F}=27.85 ; p<0.001)$. To asses which groups significantly differ Pos-Hoc Multiple comparison Hochberg's GT2 test was applied. Multiple comparison analysis showed significantly higher CHI3L1 mRNA expression in glioblastoma (grade IV) cohort compared with II and III grade gliomas $(p<0.001)$. Besides that, CHI3L1 mRNA expression in glioma grade I also showed to be significant higher than compared with grade II and III, respectively $p<0.001$ and $p=0.032$. No important $m$ RNA level difference between gliomas grades I and IV and grades II and III were observed ( $p>0.05$ ) (Fig. 1).

Next we decided to examine whether the increase of CHI3L1 mRNA level in glioblastomas is due to the promoter methylation of the CHI3L1 gene. For this purpose, methylation status of 2 sites of CHI3L1gene promoter was analyzed by MS-PCR followed by DNA bisulfite treatment. CHI3L1 methylation status showed no link between tumor malignancy grade (Chi-square test Likelihood Ratio $p>0.05$ ) or gene $m$ RNA level (Mann-Whitney test $p>0.05$ ) on both analyzed promoter sites (see Fig. 2).

\section{Correlation of CHI3L1 with clinicopathological characteristics and patient survival}

To determine the significance of the increased CHI3L1 $m$ RNA expression in glioma, we analyzed the relationship between CHI3L1 $m$ RNA levels and the clinicopathological features of glioma tumor patients. For this purpose, CHI3L1 mRNA level values obtained from the complete set of 98 different grade glioma samples have been divided into three categories: values that were lower than or equal to the 25th percentile were ranked as "low" CHI3L1 $m$ RNA level, values falling between the 25th and 75th percentile range were considered as "medium" CHI $3 \mathrm{~L} 1$ $m$ RNA level, and values that were higher than or equal to the 75th percentile were ranked as "high" CHI3L1 $m R N A$ levels. According to the RT-PCR results, "low" $m$ RNA levels of CHI3L1 were determined for 24 


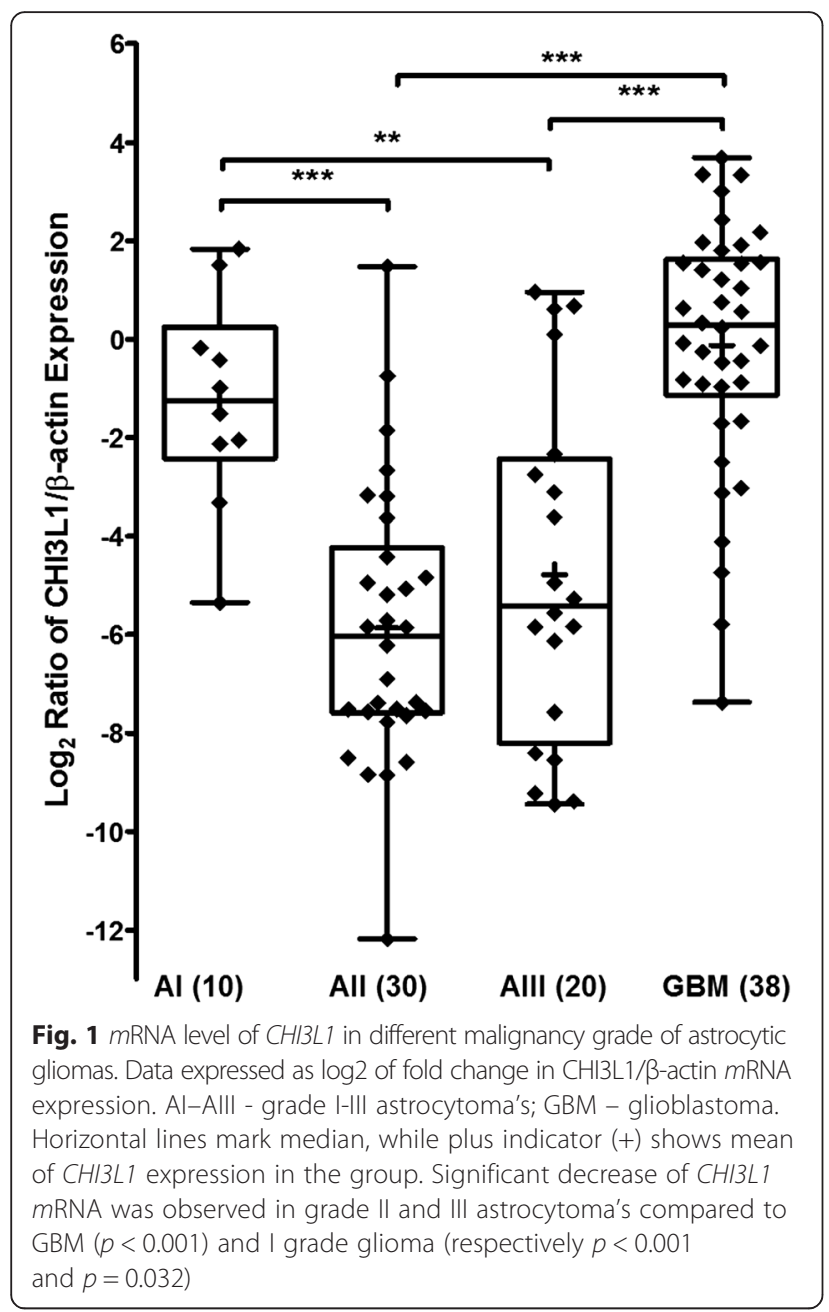

Fig. 1 mRNA level of $C H I 3 L 1$ in different malignancy grade of astrocytic gliomas. Data expressed as log2 of fold change in CHI3L1/B-actin mRNA expression. Al-All - grade I-III astrocytoma's; GBM - glioblastoma. of $\mathrm{CH} 3 \mathrm{~L} 1$ expression in the group. Significant decrease of $\mathrm{CH}$ I3L mRNA was observed in grade II and III astrocytoma's compared to GBM $(p<0.001)$ and I grade glioma (respectively $p<0.001$ and $p=0.032$
(24.5 \%), "medium" CHI3L1 levels were determined for 50 (51\%), and "high" CHI3L1 levels were determined for 24 (24.5 \%) glioma patients. As shown in Table 1, we found positive significant relationship between CHI3L1 mRNA level and high tumor malignancy $(p<0.001)$. This suggested that CHI3L1 is positively associated with glioma progression. CHIBL1 mRNA levels were also associated with patient age $(p<<0.001)$, but gen$\operatorname{der}(\mathrm{p}>0.05$, Table 1$)$.

The link between CHI3L1 expression and glioma pathological grade indicated us to check the association between patient survival and Chitinase 3-Like 1 expression. The Kaplan-Meier analysis using the log-rank test showed strong association between patient overall survival and CHI3L1 $m$ RNA expression (Log-rank test, $\chi 2=25.174$, $\mathrm{df}=2, p<0.001$ ) (see Fig. 3). Glioma patient with low CHI3L1 mRNA expression had significantly higher chance of longer survival when compared with medium and especially high CHI3L1 expression. It should be noted that these data are in the line with gene mRNA expression distribution across tumor grade - higher CHI3L1 expression was intrinsic for glioblastoma tissue. Relation between patient overall survival and CHI3L1 mRNA expression prompt us to check whether CHI3L1 mRNA level differ in WHO grade and patient survival groups. CHI3L1 mRNA expression data was divided into two groups according to patient survival $<24$ and $>24$ months (after removal of tumor) in each WHO grade. Astrocytoma grade I tumors were omitted because all grade I cases were alive on the time of analysis. Only dead subjects were included in the analysis. No statistically significant connection but tendency between CHI3L1 expression and patient survival was observed in all analysed WHO groups most likely due to small number of cases ( $t$-test: AII $p=0.493$; AIII $p=0.054$; GBM $p=0.27$ ) (see Fig. 4).

To assess the prognostic potential for independent variables (such as CHI3L1 promoter methylation, mRNA
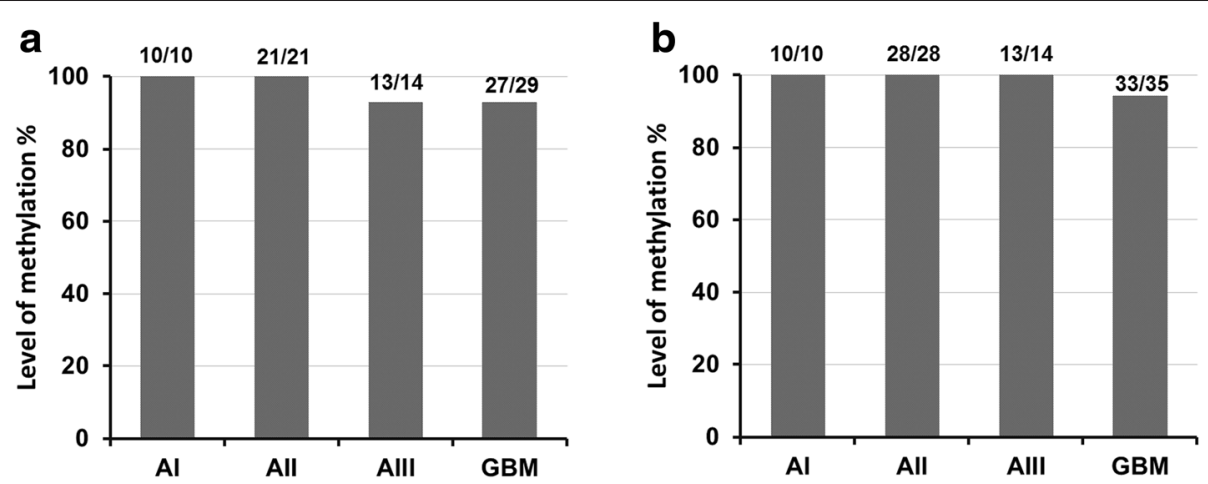

Fig. 2 Methylation analysis of CHI3L1 in different grade gliomas. a Methylation frequency analyzed with first primer set while (b) with second primer set. Both primer sets detects separate CpG dinucleotides in CHI3L1 promoter CpG island. No significant association was observed among malignancy grades and CHI3L1 methylation frequency analyzed with 1-st primer set $(x 2=3,815, \mathrm{df}=3, p=0.282)$ and 2 -nd primer set $(x 2=1,646$, $\mathrm{df}=3, p=0.649)$ 
Table 1 Association of CHI3L1 mRNA expression in human glioma tissues with different clinicopathological features

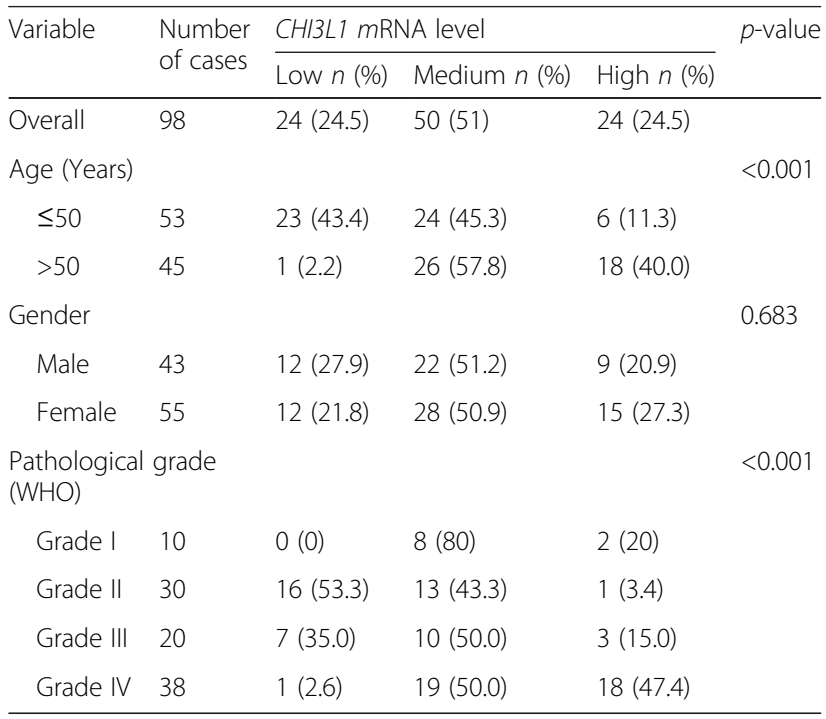

expression, astrocytoma pathological grade, age and gender) associated with patient survival univariate Cox analysis was performed. Astrocytoma grade I samples were retracted from the analysis due to patient status (all the patients were alive on the time of analysis). Univariate Cox analysis showed that CHI3L1 mRNA expression $(p<0.001)$, astrocytoma pathological grade $(p<0.001)$ and patient age $(p<0.001)$ had a significant association as independent variables with the overall survival of the patients, while CHI3L1 promoter methylation status and

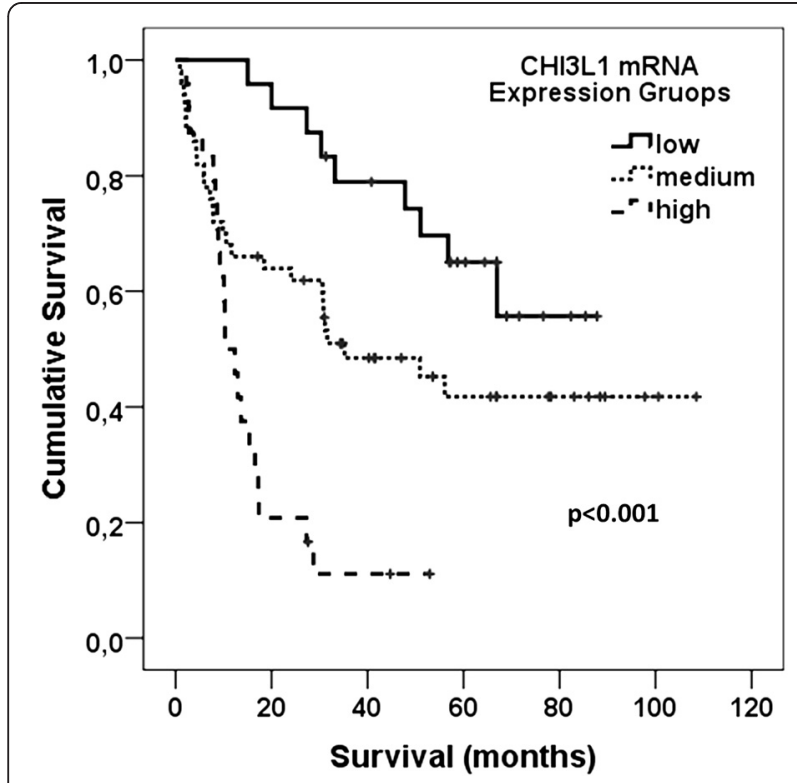

Fig. 3 Kaplan-Meier survival curves show strong secession of patient overall survival in high medium and low CHI3L1 mRNA expression groups (Log-rank test, $X 2=25.174, \mathrm{df}=2, p<0.001$ ) thus suggesting CHI3L1 impact for gliomagenesis patient gender had no significant connection $(p>0.05)$. All factors that had strong impact on survival were then evaluated jointly in multivariate Cox analysis. Astrocytoma grade II was selected as reference group when analyzing qualitative data. Multivariate analysis confirmed that astrocytoma grade, CHI3L1 mRNA expression level and patient age were considered as independent prognostic factors (see Table 2). As expected Cox analysis revealed that astrocytoma pathological grade had strong impact for patient survival as independent factor. Hazard ratio (HR) of shorter survival for grade III patient's was $2.95, p=0.017$ while for GBM almost twice higher 5.34, $p=0.002$ when compared to grade II astrocytoma. The second most important independent factor which was shown by multivariate cox analysis is the increment of CHI3L1 mRNA expression. Higher CHI3L1 mRNA level increases the risk of shorter survival for patient, HR $=1.119, p=0.038$. The age of patients was the last appointed independent factor which slightly increases the risk of shorter survival for older patient, $H R=1.033$, $p=0.022$ (see Table 2).

\section{Discussion}

One of the most dramatically induced genes in GBM is CHI3L1 [13, 14]. A wealth of clinical evidence has also revealed that elevated serum levels of CHI3L1 in GBM are positively correlated with cancer invasiveness, radioresistance, recurrence, and reduced patient survival times [15]. There are data that, CHI3L1 expression could be a prognostic predictor of glioblastoma $[5,8,16]$, although other studies have not supported this role [17]. Pelloski with colleagues used subjective score $(0,1+, 2+)$ to evaluate $\mathrm{CHI} 3 \mathrm{~L} 1$ expression and found positive correlation between CHI3L1 staining and short survival [18]. Later the same research group failed to find this correlation, but found that combined CHI3L1/EGFRvIII negative status was associated with better prognosis [19]. In other studies was found no correlation between survival and CHI3L1 mRNA level $[16,17]$. Also no correlation was found between IHC staining for CHI3L1 and patient survival [20]. Also, the literature up to date lacks crucial documentation of CHI3L1 expression with respect to tumor grade and interface with survival. Recently, a number of gene expression array studies have identified CHI3L1 to be one of the most overexpressed genes in glioblastoma when compared to low-grade glioma and normal brain $[13,14,21,22]$, but most of them were carried out with small sample number. Either several medical and inflammatory diseases have been associated with elevated serum levels of YKL-40, including polycystic ovarian sindrome [23], rheumatoid arthritis [24], diabetes mellitus [25]. These medical, inflammatory, and malignant diseases all possibly contribute to the levels of serum CHI3L1. In our study, we used real- 


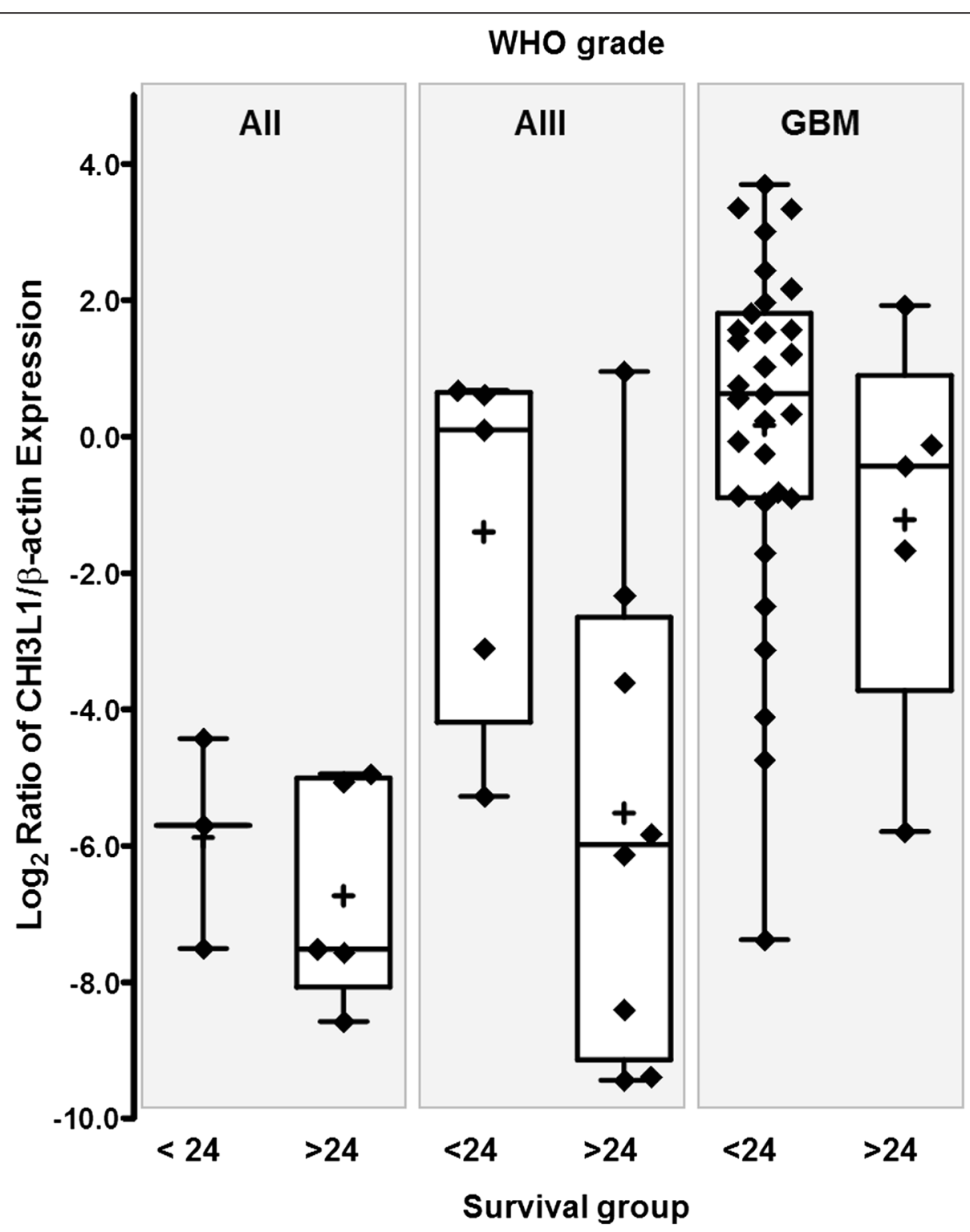

Fig. 4 Association between CHI3L1 mRNA expression and patient survival. The line inside boxes represent median, the plus symbol (+) represent the mean and the lower and upper edges of the boxes represent $25^{\text {th }}$ and $75^{\text {th }}$ percentiles, respectively. Only dead cases were included in the analysis and this has been evidently limiting factor to determine statistically significant difference between CHI3L1 expression groups in patient survival groups (t-test $p>0.05)$

Table 2 Multivariate Cox regression analysis showed that CHI3L1 mRNA level is one of the three analyzed independent variables influencing the survival of the patients

\begin{tabular}{llll}
\hline Factor & HR 95 \% Cl & B & $p$-value \\
\hline Astrocytoma grade $(\mathrm{WHO})$ & & & 0.007 \\
$\quad$ Grade III & $2.949(1.213-7.17)$ & 1.082 & 0.017 \\
$\quad$ Grade IV (GBM) & $5.342(1.814-15.73)$ & 1.676 & 0.002 \\
CHI3L1 mRNA & $1.119(1.006-1.244)$ & 0.112 & 0.038 \\
Age & $1.033(1.005-1.061)$ & 0.032 & 0.022 \\
\hline
\end{tabular}

time quantitative PCR (qRT-PCR) to measure the quantitative expression of CHI3L1 in different grade astrocytoma tissues without the influence of other malignancies or medical diseases. Our data showed that $m$ RNA expression level of CHI3L1 in glioma specimen was associated with tumour malignancy and patient overall survival. Higher mRNA level was more frequent in glioblastoma tissue as compared to grade II and III glioma. Grade I glioma also showed significantly higher CHI3L1 mRNA expression as compared to grade II and III glioma, but less than GBM. It is important to mention that grade I glioma expression profile was more similar to healthy brain (RHB) sample 
and this could indicate that expression of CHI3L1 is at very beginning stage of alteration in grade I glioma. This suggests that CHI3L1 expression shifts through gliomagenesis and is downregulated at grade II and III but upregulated in GBM. Next it would be useful to find out what molecular mechanisms are responsible for this shifting, as far as this could be very important for tumour malignancy progression. The CHI3L1 importance for gliomagenesis was showed by survival analysis. Despite grade I glioma specimen showed similar CHI3L1 expression profile to glioblastoma specimen Kaplan-Meier curves strongly separate patient with different expression level to diverse survival groups. This demonstrates that CHI3L1 mRNA expression level could be informative prognostic marker for glioma patient overall survival. Castells and colleagues reported that the expression values from only four transcripts (CHI3L1, LDHA, LGALS1, and IGFBP3) were able to distinguish two survival groups in Glioblastoma [26]. Our findings propose that mRNA expression values of CHI3L1, could be useful, not only for glioblastoma, but for lower grade astrocytoma diagnosis and prognosis too. Unlike CHI3L1 mRNA data, promoter methylation status analysis did not reveal significant relevance on gliomagenesis. Such data could be clarified in two theories: wrong selection of gene promoter sequence to analyse; or different gene regulation mechanisms than promoter methylation is intrinsic for CHI3L1. Recent discoveries found that CHI3L1 acts on glioblastoma-stem like cells (GSCs) to drive the formation of tumour vascularization and targeting CHI3L1 may compliment conventional antiangiogenic therapies to provide a substantial clinical benefit to patients with GBM [15].

\section{Conclusions}

Findings presented in our study showed that the increased mRNA level of CHI3L1 could be associated with the progression of astrocytoma and with poor patient survival not only in the glioblastoma but in the lower grade astrocytoma tumors as well. Further investigation will be required to evaluate CHI3L1 as a molecular marker for astrocytoma prognoses and for novel treatment strategies against all grade astrocytomas.

\section{Ethical standards}

Experiments described in the manuscript comply with the current laws of the country in which they were performed.

\section{Competing interests}

The authors declare that they have no competing interests.

\section{Authors' contributions}

PV, DS, AK and AT generated an idea. DS and PV extracted tissue DNA and RNA and performed bisulfite modification of DNA. DS and GS worked out MS-PCR experimental conditions for CHI3L1 analysis and together with IG did methylation experiments. GS worked for aRT-PCR experimental conditions and with RS did CHI3L1 mRNA expression experiments. GS and PV did all statistical analysis of data. PV and GS wrote a manuscript. AT gathered postoperative glioblastoma tissue and patient data. All authors read and approved the manuscript.

\section{Acknowledgements}

We heartily thank Ms. Lina Piličiauskienè and Ms. Jūrate Žeglienè from LUHS for assistance in tissue biobanking and patient clinical data gathering.

\section{Funding}

This research was funded by a grants from the Research Council of Lithuania.

Received: 11 January 2016 Accepted: 16 April 2016

Published online: 27 April 2016

\section{References}

1. Louis DN, Ohgaki H, Wiestler OD, Cavenee WK, Burger PC, Jouvet A, et al. The 2007 WHO classification of tumours of the central nervous system. Acta Neuropathol. 2007;114(2):97-109. doi:10.1007/s00401-007-0243-4.

2. Colman H, Zhang L, Sulman EP, McDonald JM, Shooshtari NL, Rivera A, et al. A multigene predictor of outcome in glioblastoma. Neuro-Oncology. 2010;12(1):49-57. doi:10.1093/neuonc/nop007.

3. Phillips HS, Kharbanda S, Chen R, Forrest WF, Soriano RH, Wu TD, et al, Molecular subclasses of high-grade glioma predict prognosis, delineate a pattern of disease progression, and resemble stages in neurogenesis. Cancer Cell. 2006;9(3):157-73. doi:10.1016/j.ccr.2006.02.019.

4. Westphal M, Lamszus K. Circulating biomarkers for gliomas. Nat Rev Neurol. 2015;11(10):556-66. doi:10.1038/nrneurol.2015.171.

5. Iwamoto FM, Hottinger AF, Karimi S, Riedel E, Dantis J, Jahdi M, et al. Serum YKL-40 is a marker of prognosis and disease status in high-grade gliomas. Neuro-Oncology. 2011;13(11):1244-51. doi:10.1093/neuonc/nor117.

6. Johansen JS, Schultz NA, Jensen BV. Plasma YKL-40: a potential new cancer biomarker? Future Oncol. 2009;5(7):1065-82. doi:10.2217/fon.09.66.

7. Chiang YC, Lin HW, Chang CF, Chang MC, Fu CF, Chen TC, et al. Overexpression of CHI3L1 is associated with chemoresistance and poor outcome of epithelial ovarian carcinoma. Oncotarget. 2015.

8. Hormigo A, Gu B, Karimi S, Riedel E, Panageas KS, Edgar MA, et al. YKL-40 and matrix metalloproteinase-9 as potential serum biomarkers for patients with high-grade gliomas. Clin Can Res. 2006;12(19):5698-704. doi:10.1158/ 1078-0432.CCR-06-0181.

9. Sakamoto F, Katakami N, Kaneto H, Yasuda T, Takahara M, Miyashita K, et al. Association of serum YKL-40 levels with urinary albumin excretion rate in young Japanese patients with type 1 diabetes mellitus. Endocr J. 2013; 60(1):73-9.

10. Kazakova M, Batalov A, Deneva T, Mateva N, Kolarov Z, Sarafian V. Relationship between sonographic parameters and YKL-40 levels in rheumatoid arthritis. Rheumatol Int. 2013;33(2):341-6. doi:10.1007/s00296012-2387-3

11. Hogdall EV, Ringsholt M, Hogdall CK, Christensen IJ, Johansen JS, Kjaer SK, et al. YKL-40 tissue expression and plasma levels in patients with ovarian cancer. BMC Cancer. 2009:9:8. doi:10.1186/1471-2407-9-8.

12. Wang D, Zhai B, Hu F, Liu C, Zhao J, Xu J. High YKL-40 serum concentration is correlated with prognosis of Chinese patients with breast cancer. PLoS One. 2012;7(12):e51127. doi:10.1371/journal.pone.0051127.

13. Nigro JM, Misra A, Zhang L, Smirnov I, Colman H, Griffin C, et al. Integrated array-comparative genomic hybridization and expression array profiles identify clinically relevant molecular subtypes of glioblastoma. Cancer Res. 2005;65(5):1678-86. doi:10.1158/0008-5472.CAN-04-2921.

14. Tanwar MK, Gilbert MR, Holland EC. Gene expression microarray analysis reveals YKL-40 to be a potential serum marker for malignant character in human glioma. Cancer Res. 2002;62(15):4364-8.

15. Shao R, Taylor SL, Oh DS, Schwartz LM. Vascular heterogeneity and targeting: the role of YKL-40 in glioblastoma vascularization. Oncotarget. 2015;6(38):40507-18. doi:10.18632/oncotarget.5943.

16. Serao NV, Delfino KR, Southey BR, Beever JE, Rodriguez-Zas SL. Cell cycle and aging, morphogenesis, and response to stimuli genes are individualized biomarkers of glioblastoma progression and survival. BMC Med Genet. 2011; 4:49. doi:10.1186/1755-8794-4-49.

17. Boisselier B, Marie Y, El Hallani S, Kaloshi G, lershov A, Kavsan V, et al. No association of $(-131 \mathrm{C}->\mathrm{G})$ variant of $\mathrm{CHI} 3 \mathrm{~L} 1$ gene with risk of glioblastoma and prognosis. J Neuro-Oncol. 2009;94(2):169-72. doi:10.1007/s11060-009-9817-4. 
18. Pelloski CE, Mahajan A, Maor M, Chang EL, Woo S, Gilbert M, et al. YKL-40 expression is associated with poorer response to radiation and shorter overall survival in glioblastoma. Clin Can Res. 2005;11(9):3326-34. doi:10.1158/1078-0432.CCR-04-1765.

19. Pelloski CE, Ballman KV, Furth AF, Zhang L, Lin E, Sulman EP, et al. Epidermal growth factor receptor variant III status defines clinically distinct subtypes of glioblastoma. J Clin Oncol. 2007;25(16):2288-94. doi:10.1200/JCO.2006.08.0705.

20. Horbinski C, Wang G, Wiley CA. YKL-40 is directly produced by tumor cells and is inversely linked to EGFR in glioblastomas. Int J Clin Exp Pathol. 2010;3(3):226-37.

21. Colin C, Baeza N, Bartoli C, Fina F, Eudes N, Nanni I, et al. Identification of genes differentially expressed in glioblastoma versus pilocytic Astrocytoma using suppression subtractive hybridization. Oncogene. 2006;25(19):2818-26. doi:10.1038/sj.onc.1209305.

22. Shostak K, Labunskyy V, Dmitrenko V, Malisheva T, Shamayev M, Rozumenko V, et al. HC gp-39 gene is upregulated in glioblastomas. Cancer Lett. 2003;198(2): 203-10.

23. Celik C, Abali R, Guzel S, Bastu E, Kucukyalcin V, Yilmaz M. Elevated circulating levels of YKL-40 are a marker of abnormal glucose tolerance in women with polycystic ovary syndrome. Clin Endocrinol. 2012;77(6):893-7. doi:10.1111/j.1365-2265.2012.04437.x.

24. Turkyilmaz AK, Devrimsel G, Kirbas A, Cicek Y, Karkucak M, Capkin E, et al. Relationship between pulse wave velocity and serum YKL-40 level in patients with early rheumatoid arthritis. Rheumatol Int. 2013;33(11):2751-6. doi:10.1007/s00296-013-2810-4.

25. Sun L, Liu JY, Li LR. Serum YKL-40 levels are associated with type 2 diabetes mellitus in patients with obstructive sleep apnea syndrome. Genet Mol Res. 2015;14(3):8919-25. doi:10.4238/2015.August.3.15.

26. Castells X, Acebes JJ, Majos C, Boluda S, Julia-Sape M, Candiota AP, et al. Robustness of equations that define molecular subtypes of glioblastoma tumors based on five transcripts measured by RT-PCR. OMICS. 2015;19(1): 41-51. doi:10.1089/omi.2014.0077.

\section{Submit your next manuscript to BioMed Central and we will help you at every step:}

- We accept pre-submission inquiries

- Our selector tool helps you to find the most relevant journal

- We provide round the clock customer support

- Convenient online submission

- Thorough peer review

- Inclusion in PubMed and all major indexing services

- Maximum visibility for your research

Submit your manuscript at www.biomedcentral.com/submit

C Biomed Central 